\title{
Impulsivity Characterization in the Roman High- and Low-Avoidance Rat Strains: Behavioral and Neurochemical Differences
}

\author{
Margarita Moreno',5, Diana Cardona',5, Maria José Gómez², Fernando Sánchez-Santed', Adolf Tobeña ${ }^{3}$, \\ Alberto Fernández-Teruel ${ }^{3}$, Leticia Campa ${ }^{4}$, Cristina Suñol ${ }^{4}$, Maria Dolores Escarabajal ${ }^{2}$, Carmen Torres $^{2}$ \\ and Pilar Flores*,I
}

'Departamento de Neurociencia y Ciencias de la Salud, Universidad de Almería, Almería, Spain; ²Departamento de Psicología, Universidad de Jaén, Jaén, Spain; ${ }^{3}$ Departamento de Psiquiatria y Medicina Legal, Instituto de Neurociencias, Universidad Autónoma de Barcelona, Barcelona, Spain; ${ }^{4}$ Instituto de Investigaciones Biomedicas de Barcelona, CSIC-IDIBAPS, CIBERESP (CS), CIBERSAM (LC), Barcelona, Spain

\begin{abstract}
The selective breeding of Roman high- (RHA) and low-avoidance (RLA) rats for rapid vs extremely poor acquisition of active avoidance behavior in a shuttle-box has generated two phenotypes with different emotional and motivational profiles. The phenotypic traits of the Roman rat lines/strains (outbred or inbred, respectively) include differences in sensation/novelty seeking, anxiety/fearfulness, stress responsivity, and susceptibility to addictive substances. We designed this study to characterize differences between the inbred RHA-I and RLA-I strains in the impulsivity trait by evaluating different aspects of the multifaceted nature of impulsive behaviors using two different models of impulsivity, the delay-discounting task and five-choice serial reaction time (5-CSRT) task. Previously, rats were evaluated on a schedule-induced polydipsia (SIP) task that has been suggested as a model of obsessive-compulsive disorder. RHA-I rats showed an increased acquisition of the SIP task, higher choice impulsivity in the delay-discounting task, and poor inhibitory control as shown by increased premature responses in the 5-CSRT task. Therefore, RHA-I rats manifested an increased impulsivity phenotype compared with RLA-I rats. Moreover, these differences in impulsivity were associated with basal neurochemical differences in striatum and nucleus accumbens monoamines found between the two strains. These findings characterize the Roman rat strains as a valid model for studying the different aspects of impulsive behavior and for analyzing the mechanisms involved in individual predisposition to impulsivity and its related psychopathologies.

Neuropsychopharmacology (2010) 35, I 198-1208; doi:I0.1038/npp.2009.224; published online 20 January 2010
\end{abstract}

Keywords: impulsivity; Roman high- and low-avoidance rats; schedule-induced polydipsia; delay-discounting task; 5-choice serial reaction time task; monoamines

\section{INTRODUCTION}

Impulsivity encompasses a range of actions that are poorly conceived, prematurely expressed, unduly risky, or inappropriate to the situation, often leading to undesirable consequences (Daruna and Barnes, 1993). High levels of impulsivity are associated with disorders including obsessive-compulsive disorder (OCD), attention-deficit hyperactivity disorder, schizophrenia, and antisocial behavior (American Psychiatric Association, 2000). Moreover, accumulating evidence suggests that the comorbidity between

*Correspondence: Dr P Flores, Departamento de Neurociencia y Ciencias de la Salud, Universidad de Almería, La Cañada de San Urbano, 04I20 Almería, Spain, Tel: + 34 9500154I2, Fax: + 34 9500 I5473, E-mail: pflores@ual.es

${ }^{5}$ These authors contributed equally to this work.

Received 10 June 2009; revised 24 November 2009; accepted 27 November 2009 impulsivity and other behavioral traits, as sensation/novelty seeking or risk taking and physiological stress responsivity (Chakroun et al, 2004; Sher et al, 2000), may share genetic factors that contribute to the vulnerability to addictive disorders (Kreek et al, 2005).

In recent years, important efforts have been made to characterize valid animal models for studying the effect of genetic background on the neural substrates of psychiatric (Annetrude et al, 2008; Gould and Gottesman, 2006; Machado-Vieira et al, 2004) and addiction disorders (Crabbe, 2002; Laakso et al, 2002). Two phenotypes with different emotional and motivational profiles have been developed through bidirectional selection and breeding of the Roman high- (RHA) and low-avoidance (RLA) rat lines/ strains (outbred or inbred, respectively), selected for rapid (RHA) vs extremely poor (RLA) acquisition of two-way active avoidance in the shuttle-box (Broadhurst and Bignami, 1965; Driscoll and Bättig, 1982) (for reviews, see 
Driscoll et al, 1990, 1998, 2009; Escorihuela et al, 1995, 1999; Fernández-Teruel et al, 1997; Giorgi et al, 2007; Steimer and Driscoll, 2005). These lines/strains show consistent differences in sensation/novelty seeking and in stress/anxiety endocrine and behavioral responses when confronted with novel environments (ie, intended to measure anxiety, emotionality, fearfulness, or novelty seeking), such as the open-field test (Aubry et al, 1995; Carrasco et al, 2008; Escorihuela et al, 1999; Gentsch et al, 1991), elevated plus maze (Escorihuela et al, 1999; Steimer and Driscoll, 2003), elevated zero-maze (López-Aumatell et al, 2009b), blackwhite box test, dark-light open-field test, dark-light hexagonal tunnel maze (Fernández-Teruel et al, 2002b; Steimer and Driscoll, 2005), and hole-board test (Escorihuela et al, 1999; Fernández-Teruel et al, 1992). In all of these situations and in fear-conditioning procedures (eg, fear-potentiated startle; López-Aumatell et al, 2009a), RLA rats show increased anxiety or fear, a passive coping style, and increased stress responses by hypothalamus-pituitaryadrenal (HPA) axis activation (Carrasco et al, 2008; Steimer and Driscoll, 2003). RHA rats tend to be novelty/sensation seekers with relatively low HPA axis activation (Driscoll et al, 1998, 2009). Moreover, these differences occur with individual differences in vulnerability to drug addiction, manifested as behavioral and neurochemical responses to different drugs of abuse such as ethanol, morphine, and cocaine (Fattore et al, 2009; Giorgi et al, 2007). Abundant evidence implicates dopaminergic mechanisms in these differences. Compared with RLA rats, RHA rats show different dopaminergic responses: activation in the mesocortical dopaminergic pathway by stressors and anxiogenic drugs (Giorgi et al, 2003); more rapid baseline turnover rate of dopamine (DA) in the caudate nucleus (Driscoll et al, 1990); and more intense stereotypy in response to acute challenge with the DA receptor agonist apomorphine (Giménez-Llort et al, 2005).

In spite of the role of impulsivity as a principal symptom of most of the above mentioned psychiatric disorders (Evenden and Ryan, 1996), only one study has investigated impulsivity differences in these two rat lines (Zeier et al, 1978). During the acquisition of a DRL-20 task, RHA rats showed more irrelevant lever-pressing behavior than their RLA counterparts, suggesting reduced inhibition of impulsive behavior. However, there have been no other studies that have systemically investigated differences in impulse control between RHA-I and RLA-I rat strains.

In this study, we investigated possible differences in impulsivity between the RHA-I and RLA-I inbred strains to assess their potential as a valid model for studying the basis of impulsivity, dividing this multifaceted trait (Evenden, 1999) into two testable categories: (1) impulsive choice, measured by intolerance of gratification delay in the delaydiscounting task (Cardona et al, 2006; Winstanley et al, 2004, 2006), and (2) impulsive behavior, measured by poor inhibitory control of inappropriate responses in a fivechoice serial reaction time (5-CSRT) task (Robbins, 2002; Winstanley et al, 2006). We also examined possible between-strain differences on schedule-induced polydipsia (SIP) task that has been suggested as a model of OCD (Platt et al, 2008; Rosenzweig-Lipson et al, 2007; Woods et al, 1993; Woods-Kettelberger et al, 1996). As impulsive behaviors have been related to monoaminergic dysfuctions
(Winstanley et al, 2006), we assessed basal neurochemical function in the striatum and nucleus accumbens of RHA-I and RLA-I rats.

\section{MATERIALS AND METHODS}

\section{Animals}

Fourteen RHA-I and 14 RLA-I inbred female rats, between 90 and 120 days of age at the start of the experiments, were used. They were bred at the Autonomous University of Barcelona (Spain) from colonies established in 1997 (Driscoll et al, 1998; Escorihuela et al, 1997, 1999). Although the literature refers to gender differences in the expression of impulsive behavior, we used female rats in the present experiments because the behavioral traits that distinguish the Roman lines have been consistently confirmed in both sexes across several laboratories and along different generations (Aguilar et al, 2003; Corda et al, 2005; Ferre et al, 1995; Rosas et al, 2007).

Animals were housed $3 /$ cage $(50 \times 15 \times 25 \mathrm{~cm})$ at $22{ }^{\circ} \mathrm{C}$ with $0800-2000 \mathrm{~h}$ light-dark cycle, water available, and deprived to $85 \%$ of ad libitum feeding weight through daily feedings of lab chow approximately $30 \mathrm{~min}$ after each experimental session. All testing was carried out between 0800 and $1400 \mathrm{~h}$. All procedures were performed in accordance with Spanish Royal Decree 1201/2005 on the protection of experimental animals and with the European Communities Council Directive (86/609/EEC).

The order of training and screening was as follows: SIP, delay-discounting, and 5-CSRT task. Each task commenced at least 2 weeks after the previous one, and interference among tasks was unlikely because each involved a different type of operant response for food reward.

\section{Apparatus: Operant Chambers}

We conducted the tests in seven standard operantconditioning chambers (MED Associates) 32-cm long $\times 25-\mathrm{cm}$ wide $\times 34-\mathrm{cm}$ high, with stainless-steel grid floors. A detailed description of the apparatus has been provided previously for SIP and delay-discounting task (Cardona et al, 2006; López-Grancha et al, 2008). The same chambers were also used in the 5-CSRT task, with an array of five contiguous square holes $(2.5 \mathrm{~cm}), 2.2-\mathrm{cm}$ deep and $2 \mathrm{~cm}$ above floor level at the back panel. These apertures had photocell beams at the entrance and a yellow stimulus light for the nose-poke response. The scheduling and recording of experimental events was controlled by a Med PC computer and commercial software (Cibertec SA, Spain).

\section{Experimental Procedures}

Schedule-induced polydipsia. Over two successive days, a water-ingestion test was given (baseline). Sixty pellets were placed together, and the amount of water each rat consumed in $60 \mathrm{~min}$ was measured. After one adaptation day, the animals were exposed to a fixed time $60 \mathrm{~s}$ (FT- $60 \mathrm{~s}$ ) schedule of food pellet presentation during 60-min sessions. Water bottles containing $100-\mathrm{ml}$ fresh water were provided immediately before each session. The following measures were recorded for each rat: (a) total amount of water $(\mathrm{ml})$ 
removed from the bottle, (b) total number of licks, and (c) and total number of nose-pokes (see López-Grancha et al, 2008, for more details).

Delay-discounting task. In this task, response on one lever (A) produced a one-pellet reward; response on the other lever (B) produced a four-pellet reward. Each session consisted of 60 trials, 30 forced choice and 30 free choice in which both levers were presented. Illumination of the house light and tray light signaled the start of each trial. A nose-poke response was required in the food pellet receptacle to initiate lever presentation. Failure to make the response or to depress the lever within $10 \mathrm{~s}$ terminated the trial. When either lever was pressed, the house light turned off, the 'clicker' sounded, and both levers were retracted. Food reward was then delivered, either almost immediately or after a delay. The experiment was divided into five sets of five sessions. During the first set, both levers had a delay of $0 \mathrm{~s}$. The delay to reward A remained constant at $0 \mathrm{~s}$ throughout the experiment, whereas the delay to reward B increased to $10 \mathrm{~s}$ in set $2,20 \mathrm{~s}$ in set 3 , and $40 \mathrm{~s}$ in set 4 . Set 5 was identical to set 1 : levers A and B were both $0 \mathrm{~s}$. Only data from the last two sessions per set were analyzed (see Cardona et al, 2006, for more details).

5-CSRT task. Animals were required to respond to brief flashes of light presented randomly in one of five spatial locations (Carli et al, 1983). Pre-training and training were based on previous procedures with subtle modifications (Robbins, 2002; van Gaalen et al, 2006). Rats were habituated to the apparatus over 2 days for $20 \mathrm{~min} / \mathrm{session}$. In these sessions, the house light was switched on and 20 food pellets were placed in the magazine and two in each open aperture.

Pre-training, phase 1. All cue lights and house light were switched on throughout the session. A nose-poke in one of the five holes elicited a food pellet delivery. A session ended after delivery of 100 pellets or after $30 \mathrm{~min}$. Animals were trained until all rats reliably earned 100 pellets within $30 \mathrm{~min}$.

Pre-training phase 2. Rats were trained to detect brief flashes of light (stimulus duration, SD $30 \mathrm{~s}$ ) presented in any one of the five spatial locations (in pseudorandom order) with the house light switched on during the entire session. A response into the illuminated (correct) hole elicited a food pellet delivery in the food magazine (concurrently with its illumination), and the stimulus light was extinguished. The collection of the pellet from the food magazine started a new trial. Responses into the other holes were counted but had no further consequences, with the opportunity to make a response in the correct aperture within the $30 \mathrm{~s}$ of SD. Animals were tested until all animals earned 100 pellets within $30 \mathrm{~min}$. After these pre-training phases the 5-CSRT task training commenced.

Training. Animals were on the 5-CSRT task to criterion performance (accuracy $>80 \%$, omissions $<20 \%$ ), with SD of $1 \mathrm{~s}$. Each test session commenced with the illumination of the chamber by the house light and of the food magazine in which a food pellet was delivered. The collection of this pellet from the feeder started the first trial, the next trials were self-initiated by a nose-poke response in the food magazine; then the light of the food magazine was extinguished and a fixed inter-trial interval (ITI) of $5 \mathrm{~s}$ started. At the end of the ITI, a visual stimulus with 1-s duration (SD) was presented in a random location of the apertures at the rear. Responses in this aperture within $5 \mathrm{~s}$, the limited hold (LH), were recorded as correct responses and were rewarded by a food pellet delivery in the magazine feeder with its illumination. Response errors were omissions (failure to respond to the stimulus within the LH), errors of commission (incorrect responses made to the wrong location), and premature responses (responses made before the presentation of the visual stimulus in any of the five apertures during ITI). These response errors were all punished with a 5-s period of darkness (time-out), during which no food was delivered. An additional response in an aperture after a correct response and before the food collection was recorded as a perseverative response and had no punishment of time-out period; the collection of the pellet from the feeder started the next trial. A response in the food magazine after the delivery of food or after a time-out period initiated the next trial; a response in the food magazine after a premature response restarted the same trial. Each session terminated after 100 trials or after $30 \mathrm{~min}$. To facilitate acquisition of the 5-CSRT task, SD of target stimuli was progressively shortened from 6 to $1 \mathrm{~s}$ over eight training stages. Thus, in stage $1, S D=6 \mathrm{~s}$ with $\mathrm{LH}=6 \mathrm{~s}$ and ITI $=5 \mathrm{~s}$, while SDs of stages 2 through 8 were $5,2.5,2,1.75,1.5,1.25$, and $1 \mathrm{~s}$, respectively (LH and ITI $=5 \mathrm{~s}$ ). Animals advanced through stages by completing at least 50 correct trials with $80 \%$ accuracy and $<20 \%$ omissions. Owing to problems of RLA-I to reach the criterion of $<20 \%$ omissions, rats were allowed to progress with $<30 \%$ omissions and stable $80 \%$ accuracy for at least three consecutive sessions.

The recorded measures were as follows:

Accuracy: percentage of correct trials (correct trials/ correct trials + incorrect trials) $\times 100$.

Correct responses: nose-poke responses during the LH period in the same aperture as the stimulus light presentation.

Incorrect responses: nose-poke responses during the $\mathrm{LH}$ period in a different aperture from the one in which the stimulus light was presented.

Omissions: percentage of omissions among total number of trials (number of omissions/correct responses + incorrect responses + omissions) $\times 100$

Premature responses: number of responses before the visual target presents in the apertures during the ITI periods.

Perseverative responses: number of repetitive responses at the response apertures after a correct response and before collection of the food reward.

Latencies (in seconds) to: correct response from stimulus presentation to a correct nose-poke response; incorrect response from stimulus presentation to an incorrect nosepoke response; and to reward to collect a food pellet in the magazine after a correct response.

\section{Brain Monoamine Analyses}

One day after the last behavioral task, eight animals of each strain were rapidly decapitated using a guillotine. Brains were quickly removed, frozen, and stored at $-80{ }^{\circ} \mathrm{C}$ until 
preparation (Moreno et al, 2008). For brain tissue preparation, the samples were thawed enough to allow dissection of the striatum and nucleus accumbens. These were weighed and homogenized in $0.4 \mathrm{~N}$ perchloric acid with 0.1 metabisulfite, $0.01 \%$ EDTA, and $1 \mathrm{mg} / \mathrm{ml}$ cysteine. The homogenates were centrifuged at 15000 r.p.m. for $20 \mathrm{~min}$ at $4{ }^{\circ} \mathrm{C}$, and supernatants were collected and frozen at $-80^{\circ} \mathrm{C}$ until biochemical analyses for determining the levels of norepinephrine (NE), DA, 5-HT, and their metabolites, dihydroxyphenylacetic acid (DOPAC), homovanillic acid (HVA), and 5-hydroxy-3-indolacetic acid (5-HIAA). These were measured using reverse-phase high performance liquid chromatography with electrochemical detection $(+0.7$ $\mathrm{V})$. The mobile phase, containing (in $\mathrm{mM}$ ) $\mathrm{KH}_{2} \mathrm{PO}_{4}, 100$; Na2-EDTA, $0.1 ; \mathrm{PICB}_{8}, 2.06$; plus $16 \%$ methanol, adjusted to $\mathrm{pH} 2.65$ with orthophosphoric acid, was delivered at $1 \mathrm{ml} /$ min. Monoamines were separated on a $5-\mu \mathrm{m}$ particle size column at $30^{\circ} \mathrm{C}$ (Phenomenex $\mathrm{C} 2510 \times 0.46 \mathrm{~cm}$, Micron Analítica SA, Spain).

\section{Data Analysis}

Behavioral data were analyzed using repeated measures analysis of variance (ANOVA), with Strain (RHA-I and RLA-I) as the between-subject factor and Sessions or Delay as the within-subject factor. We used one-way ANOVA to evaluate behavioral parameters for the 5-CSRT task and the monoamine data, with Strain (RHA-I and RLA-I) as the between-subject factor. When appropriate, post hoc comparisons were made using the Newman-Keuls test. To asses the integration between impulsive measures and neurochemistry, Spearman rank correlations were calculated in both groups. All analyses were computed using the Statistica software package. Statistical significance was set at $P<0.05$.

\section{RESULTS}

\section{Schedule-Induced Polydipsia}

Figure 1 shows the mean water intake (a) and the mean total licks (b) for each strain during the acquisition and maintenance of SIP. Rats developed SIP after being exposed to the FT 60-s schedule of food presentation. During the baseline period, RHA-I and RLA-I rats drank a mean of $7.8 \pm 0.9$ and $8.7 \pm 1.3 \mathrm{ml}$, respectively. The mean intake of water for these groups during the last 5 days of the experiment was $20.9 \pm 1.6$ and $12.3 \pm 0.7 \mathrm{ml}$ for RHA-I and RLA-I, respectively (Figure 1a). This SIP acquisition was also observed by the increase in the number of licks (b). During the first 3 days, RHA-I and RLA-I rats licked a mean of $1588.3 \pm 160.3$ and $1018 \pm 123.3$, respectively. The mean total licks for these groups averaged across the last 5 days of the experiment were $3827.9 \pm 435.1$ and $1514.2 \pm 148.1$ for RHA-I and RLA-I, respectively (Figure $1 \mathrm{~b}$ ). Regarding the high drinkers (HD) and low drinkers (LD) assessment on SIP, we found a high correspondence between RHA-I and HD, as well as between RLA-I and LD (data not shown).

ANOVA revealed significant differences between RHA-I and RLA-I in water intake (a) $(\mathrm{F} 1,26=14.69 ; P<0.001)$ and total licks (b) $(\mathrm{F} 1,26=25.01 ; P<0.001)$. Session effects were significant in both measures: water intake (a)
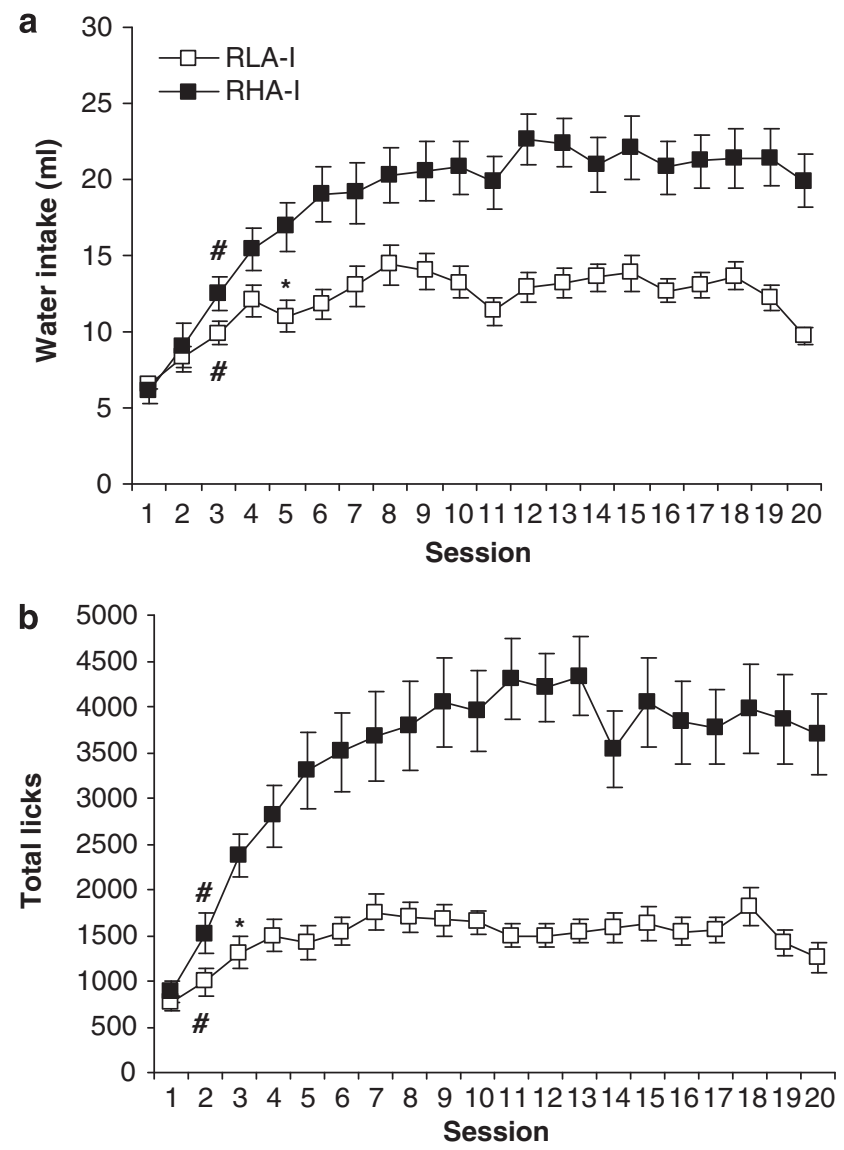

Figure I The mean $( \pm$ SEM) water intake (panel a) and total licks (panel b) in FT $60 \mathrm{~s}$ across 20 sessions of SIP. (*) Statistical analyses indicate significant differences between RHA-I and RLA-I from that session onward. (\#) Significant differences from session I.

$(\mathrm{F} 19,492=32.00 P<0.001)$ and in total licks $(\mathrm{b})(\mathrm{F} 19,492=$ 18.98; $P<0.001)$. Interaction between Sessions and Strain was also significant $(\mathrm{F} 19,492=6.72 ; P<0.001)$ in water intake (a) and in total licks (b) $(\mathrm{F} 19,492=7.64 ; P<0.001)$. Post hoc analysis indicated that the FT 60-s schedule of food delivery induced different drinking rates across the 20 test sessions in both groups. Differences in water intake between RHA-I and RLA-I animals were evident from session 5, and from session 3 in total licks. A main effect of Session (F1, $26=4.77 ; P<0.001)$ emerged for the nosepoke measure, but not for Strain $(\mathrm{F} 19,492=0.63 ; P>0.05)$. The RHA-I rats reached a higher asymptotic level on SIP, evidenced by the increased water intake and number of total licks in the first sessions and a higher rate of licks at the end of training.

\section{Delay-Discounting Task}

Figure 2 shows the mean number of choices of the large reward during the delay-discounting task. All rats chose the large reward in almost every trial when the delay to the large reward was $0 \mathrm{~s}$. ANOVA showed a Delay effect (F3, 78 $=81.80 ; P<0.001)$ : as the delay to the large reward increased, the preference of all the rats shifted toward the smaller but more immediate reward. A Strain effect was also observed $(\mathrm{F} 1,26=4.51 ; P<0.001)$, and the interaction 


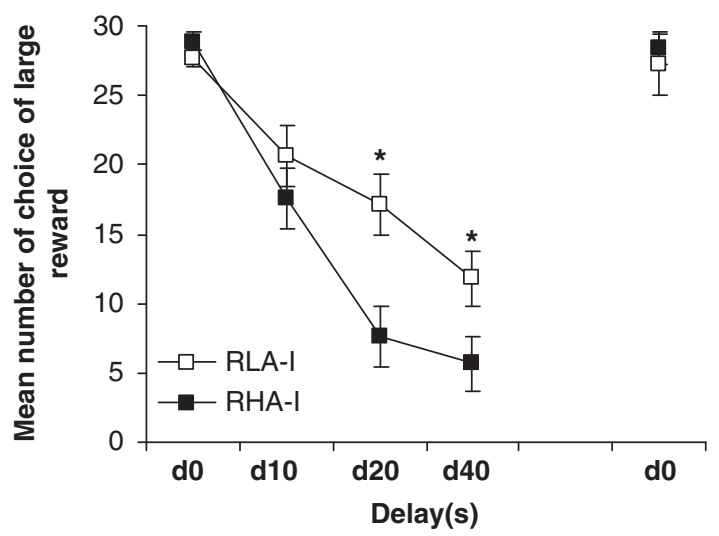

Figure 2 The mean $( \pm$ SEM) number of choices of the large reward on the delay-discounting task between strains. (*) Statistical analyses indicate significant differences in delay between RHA-I and RLA-I.

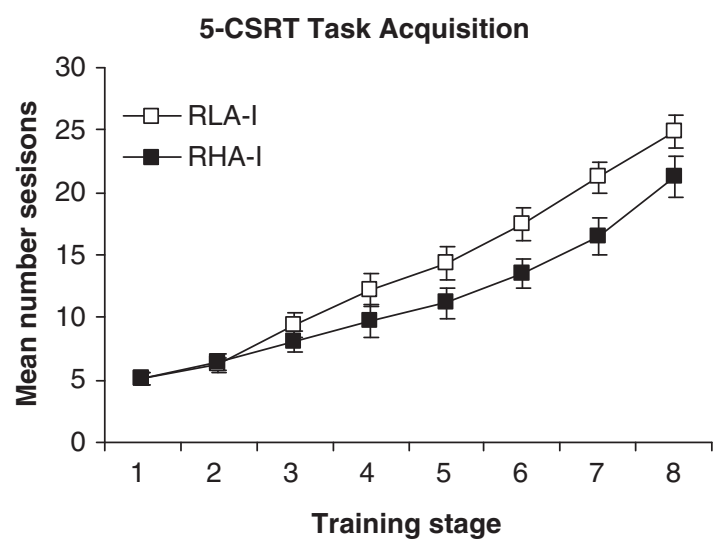

Figure 3 5-CSRT task acquisition of RHA-I and RLA-I rats. The data are the cumulative number of sessions \pm SEM required to reach each training stage (see Materials and methods section for details on the training procedure).

between Strain and Delay was significant $(\mathrm{F} 3,78=5.87$; $P<0.001)$. Post hoc analyses indicated significant differences for 20 -s and 40 -s delays $(P<0.05)$. Finally, when the delay to the large reward was removed in the final set of sessions, all animals returned to choosing the large reward in almost all trials. Taken together, these results show that the RHA-I rats showed a significantly greater preference for small immediate rewards $v s$ large delayed rewards compared with RLA-I rats (ie, they showed increased choice impulsivity).

\section{5-CSRT Task}

Figure 3 shows the effect of rat strain on the acquisition of the 5-CSRT task. RLA-I rats required an average of $24.9 \pm 1.3$ sessions to achieve criterion performance $(\mathrm{SD}=1)$ on the 5 -CSRT task, compared with $21.3 \pm 1.6$ sessions for RHA-I rats. The ANOVA revealed no significant differences between RHA and RLA in number of sessions $(\mathrm{F} 1,21=3.75 ; P>0.05)$. Interaction between Sessions and Strain was significant $(\mathrm{F} 19,147=3.11$; $P<0.05)$. Post hoc analysis indicated significant differences between Strain $\times$ Sessions from training stage 4 until the last training stage, 8 . This difference in number of sessions a

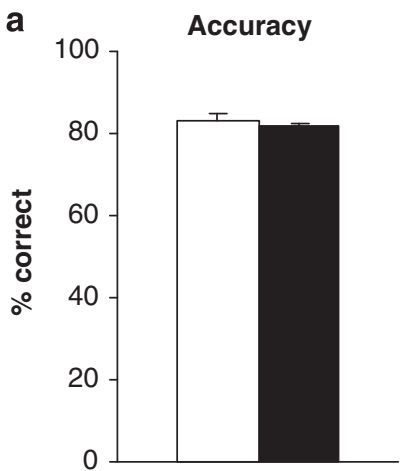

b Omissions
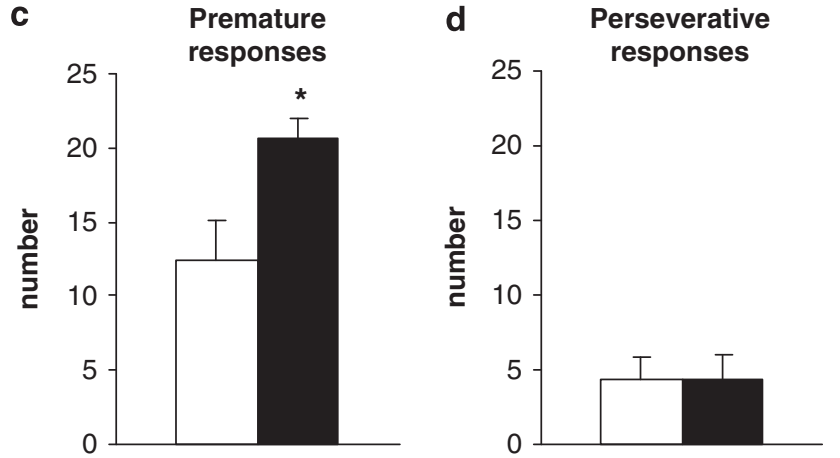

Figure 4 Performance of RHA-I and RLA-I on the 5-CSRT task $(S D=1)$ : percentage of accuracy (a), percentage of omissions (b), number of premature responses (c), and number of perseverative responses (d). RHA-I rats made more premature responses, while low-avoidance rats made a significant percentage of omissions. Data are means \pm SEM; (*) statistical analyses indicate significant differences between RHA-I and RLA-I.

to reach the training stage was the result of problems the RLA-I animals had in meeting the criterion of $<20 \%$ of omissions required to progress to the next stage.

Figure 4 shows the performance in different parameters of the 5-CSRT task $(S D=1)$ by RLA-I and RHA-I rats. Oneway ANOVA revealed no differences in accuracy between RLA-I and RHA-I animals (Figure 4a). In contrast, compared with RHA-I rats, RLA-I rats showed a significantly increased percentage of omission errors out of the total number of trials $(\mathrm{F} 1,21=36.50 ; P<0.001)$ (Figure $4 \mathrm{~b}$ ). The increased omissions among RLA-I animals were observed across the training stages. In contrast, compared with the RLA-I group, the RHA-I animals had a significantly higher number of premature responses, defined as the number of responses before the visual target occurs $(\mathrm{F} 1,21=8.80$; $P<0.05)$ (Figure 4c). No between-strain differences emerged in perseverative responses $(\mathrm{F} 1,21=0.0002 ; P>0.05)$ (Figure $4 \mathrm{~d}$ ). Compared with the RHA-I group, the latencies were slower in RLA-I rats for all parameters (Figure 5), that is, from stimulus presentation to a correct response $(\mathrm{F} 1,21=11.53 ; P<0.05)$; to an incorrect response $(\mathrm{F} 1,21=14.31 ; P<0.05)$; and to collect the reward in the magazine after a correct response $(\mathrm{F} 1,21=10.45 ; P<0.05)$.

\section{Monoamine Analysis}

RLA-I and RHA-I rats showed differences in monoamine systems in the striatum and nucleus accumbens under basal 


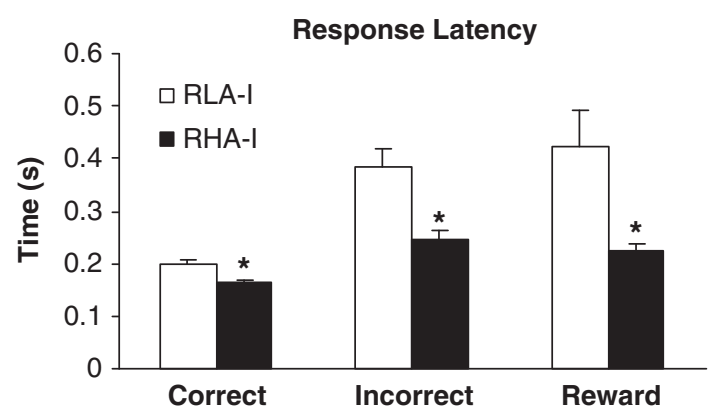

Figure 5 Latency to correct response, incorrect response, and reward RHA-I and RLA-I on 5-CSRT task $(S D=1)$. Data are means \pm SEM; (*) statistical analyses indicate significant differences between RHA-I and RLA-I.

Table I Monoamine Concentration Levels (pmol/mg tissue) in Striatum and Nucleus Accumbens (NAc) in RHA-I and RLA-I Rats

\begin{tabular}{|c|c|c|c|c|}
\hline & \multicolumn{2}{|c|}{ Striatum } & \multicolumn{2}{|c|}{ NAc } \\
\hline & RLA-I & RHA-I & RLA-I & RHA-I \\
\hline $5-\mathrm{HT}$ & $1.81 \pm 0.10$ & $2.84 \pm 0.15 *$ & $2.74 \pm 0.22$ & $3.51 \pm 0.26 *$ \\
\hline $5-\mathrm{HIAA}$ & $1.64 \pm 0.08$ & $2.58 \pm 0.09 *$ & $1.79 \pm 0.14$ & $2.48 \pm 0.18 *$ \\
\hline NE & $0.29 \pm 0.02$ & $0.57 \pm 0.08 *$ & $3.07 \pm 0.28$ & $6.03 \pm 0.76 *$ \\
\hline DA & $51.20 \pm 2.84$ & $49.22 \pm 3.46$ & $16.86 \pm 1.44$ & $16.69 \pm 2.04$ \\
\hline DOPAC & $7.58 \pm 0.50$ & $6.84 \pm 0.57$ & $4.45 \pm 0.38$ & $3.60 \pm 0.55$ \\
\hline HVA & $3.30 \pm 0.27$ & $2.84 \pm 0.21$ & $1.53 \pm 0.16$ & $1.26 \pm 0.12$ \\
\hline
\end{tabular}

Data are means \pm SEM; $(*)$ statistical analyses indicate significant differences between RHA-I and RLA-I. a

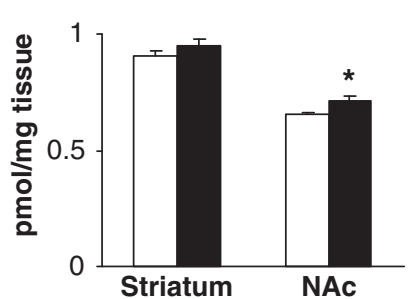

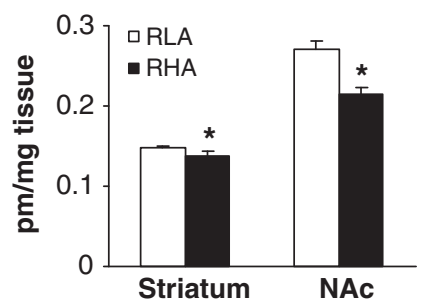

b Ratio DOPAC+HVA/DA

Figure 6 DOPAC + HVA/DA and 5-HIAA/5-HT turnover ratios (pmol/ $\mathrm{mg}$ tissue) in striatum and nucleus accumbens (NAC) in RHA-I and RLA-I rats. Data are means $\pm \mathrm{SEM} ;(*)$ statistical analyses indicate significant differences between RHA-I and RLA-I.

conditions. In the striatum and nucleus accumbens, levels of 5 -HT $\quad(\mathrm{F} 1,14=28.18 ; P<0.001 ; \mathrm{F} 1,14=5.8 ; P<0.05$, respectively), 5-HIAA (F1, $14=6.7 ; P<0.001 ; \mathrm{F} 1,14=10.76$; $P<0.05)$, and $\mathrm{NE}(\mathrm{F} 1,14=12.67 ; P<0.05 ; \mathrm{F} 1,14=14.83$; $P<0.05)$ were significantly higher in the RHA-I compared with RLA-I rats (Table 1). An increased 5-HIAA/5-HT turnover ratio in nucleus accumbens $(\mathrm{F} 1,14=6.10 ; P<0.05)$ was also observed in RHA-I rats. No significant changes were found in the striatum (Figure 6a).

Although no significant between-strain differences emerged in the levels of DA and its metabolites, DOPAC and HVA,
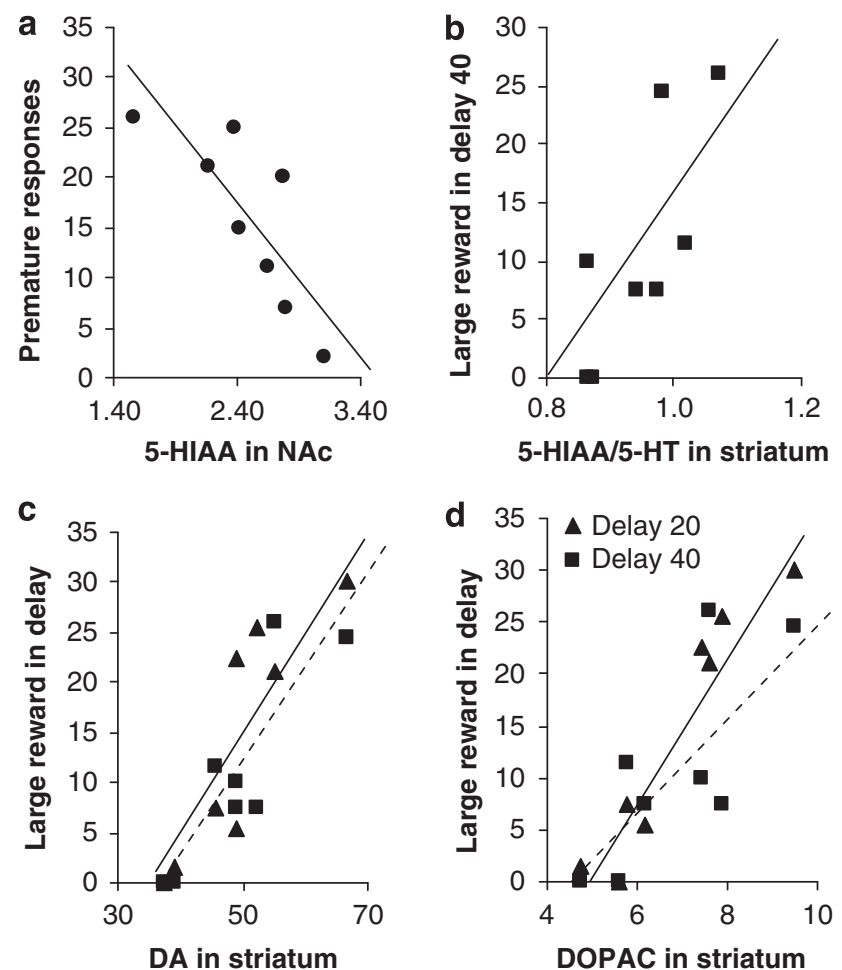

Figure 7 Correlations for RHA-I rats between neurochemical measures and impulsivity assessment. Premature responses on the 5-CSRT task and levels of 5-HIAA in NAc (a), number of choices of the large reward on the delay-discounting task (delay 40) and 5-HIAA/5-HT levels in striatum (b), number of choices of the large reward on the delay-discounting task, delay 20 (-) and delay 40 (-- -) and levels of DA and DOPAC in striatum (c, d).

we did identify a significant decrease in (DOPAC + HVA)/DA turnover ratio in the RHA-I in both the striatum and nucleus accumbens (F1, $14=5.24 ; P<0.05 ; \mathrm{F} 1,14=12.44 ; P<0.05$, respectively) (Figure $6 \mathrm{~b}$ ).

When comparing individual data of impulsivity measures with neurochemical findings, we found significant correlations for RHA-I, but not for the RLA-I group (Figure 7). In RHA-I rats, there was a negative correlation between premature responding in the 5-CSRT task with 5-HIAA metabolite $(r=-0.90, P<0.05)$ in nucleus accumbens. In contrast, there was positive correlation between choice of large reward in delay-discounting task and 5-HIAA/5-HT turnover ratio (delay $40, r=0.72, P<0.05$ ), and DA (delay 20, $r=0.90$; delay $40, r=0.78, P<0.05$ ) and DOPAC (delay $20, r=0.93, P<0.001$; delay $40, r=0.67, P=0.06)$ in striatum.

\section{DISCUSSION}

We have examined the impulsive profile of psychogenetically selected RHA-I and RLA-I female rats in different experimental paradigms frequently used for studying impulsive behavior. We also examined between-strain differences in SIP (a suggested model for OCD). RHA-I rats showed a higher choice impulsivity in the delaydiscounting task, poor inhibitory control as shown by increased premature responses in the 5-CSRT task, and increased acquisition of the SIP task compared with RLA-I 
rats. In addition, RHA-I and RLA-I rats showed significant differences in monoamine measures in the striatum and nucleus accumbens under basal conditions, with significant relations for RHA-I rats between impulsivity assessment and neurochemical findings.

\section{Increased Adjunctive Drinking Acquisition in RHA-I Rats}

In the SIP task, despite the development of SIP in both rat strains, the RHA-I rats showed stronger acquisition of the adjunctive behavior. The RHA-I rats acquired a higher asymptotic level than the RLA-I rats, showing an increased level of water intake and number of total licks from the first sessions until the end of training.

As a result of its characteristics of 'excessiveness' and 'persistence', SIP (Falk, 1961) has been suggested as a model of OCD (Platt et al, 2008; Woods et al, 1993). Moreover, the SIP model seems to be sensitive to detect susceptibilities in individuals to addictive behavior (Gilpin et al, 2008; Mittleman et al, 2003; Toscano et al, 2008) and bingeeating behavior (Moreno et al, 2009). Curiously and relevant to this work, these disorders also share impulsivity as one of the main symptoms (American Psychiatric Association, 2000). In fact, the development of SIP has revealed marked individual differences among animals (Cardona et al, 2006; López-Grancha et al, 2008; Mittleman and Valenstein, 1984, 1985; Piazza et al, 1993), allowing their separation into high (HD) and low drinkers (LD) populations, according to whether their rates of drinking are above or below the median. Our findings show a high correspondence of RHA-I and HD and between RLA-I and LD. Therefore, our present results point to SIP as a sensitive task for separating specific populations, which could be of potential interest as an animal model for analyzing the neurobehavioral basis of vulnerability to several psychopathologies related to impulsivity.

\section{Higher Choice Impulsivity and Poor Inhibitory Control in RHA Rats}

RHA-I rats showed a high impulsivity profile in two distinct forms of measures related to the impulsivity trait: the delaydiscounting and the 5-CSRT task. The delay-discounting paradigm is a widely used task to measure impulsive choice. This task evaluates whether animals prefer a smaller but immediate reward or a larger but delayed reward (Evenden and Ryan, 1996; Winstanley et al, 2004), with impulsive choice defined as the selection of the smaller immediate reward. We found that RHA-I rats made fewer choices of a large reward when the delay was increased from 0 to 20 and $40 \mathrm{~s}$. Thus, RHA-I rats prefer an immediate reward and show marked choice impulsivity compared with RLA-I rats. Other studies have found strain differences in impulsivity with the same approach, such as greater impulsivity in Lewis rats compared with Fischer 344 rats (Anderson and Woolverton, 2005). This finding appears to agree with the increased susceptibility to addiction (and effects of addictive drugs) of Lewis $v s$ Fischer 344 rats (Kosten and Ambrosio, 2002, for review; Sánchez-Cardoso et al, 2009) and is also in line with similar findings in RHA vs RLA rats (eg, Fattore et al, 2009; Fernández-Teruel et al, 2002a) and the current results.

The 5-CSRT task measures parameters related to attention and inhibitory control of impulsive actions, such as premature responses (Robbins, 2002; Robinson et al, 2009; Winstanley et al, 2006). In spite of the absence of betweenstrain differences in accuracy, RHA-I rats showed an increased number of premature responses compared with RLA-I rats. This measure is an index of impulsive behavior because it occurs when inhibitory control of highly prepotent responses has been maladaptively lost (Robbins, 2002). Consequently, RHA-I animals showed higher impulsive behavior than RLA-I rats in the 5-CSRT task.

Moreover, the increased error omissions observed in RLA-I rats might hypothetically reflect sensory, motor, or motivational factors (Robbins, 2002). However, the concordant lack of changes in accuracy and the increased response latency in RLA-I rats point to motivational/ emotional factors (rather than impairments in attentional processes) as a possible mechanism underlying strain differences for this parameter. Compared with RHA-I rats, RLA-I rats are characterized by increased anxiety/fear levels, enhanced (hormonal and behavioral) sensitivity to stressful situations, and shows of characteristic passive coping strategies (eg, freezing behavior) when faced with conflict or stressful situations (Carrasco et al, 2008; Driscoll et al, 2009; López-Aumatell et al, 2009a,b; Steimer and Driscoll, 2003, 2005). In this sense, it appears likely that the demanding (ie, stressful) conditions of the 5-CSRT task could have influenced motivational/emotional aspects in RLA-I rats, possibly leading to their longer response latencies and increased errors of omissions.

The differences in premature responding reported here are not the result of artefacts unrelated to impulsivity; recent work on Lister-Hooded rats selected for differences in impulsive behavior, showed similar baselines values of premature responding on the 5-CSRT task to those showed by RHA-I and RLA-I animals in the present experiment (Robinson et al, 2009). In fact, premature responses in both groups were above and below, respectively, the normal average for Wistar and Sprague-Dawley rats (Moreno et al, unpublished data from our laboratory; Semenova and Markou, 2007; Semenova et al, 2007; van Gaalen et al, 2006).

\section{Neurochemical Differences in RHA vs RLA: Association with Impulsivity Phenotype}

Impulsive behaviors have been related to monoaminergic dysfunction in limbic brain areas (Cardinal et al, 2001). Moreover, the relationship between monoamine levels and impulsivity depends on the particular impulsive behavior evaluated (Winstanley et al, 2006, for review). Convergent evidence suggests that monoaminergic (at least DA and 5HT) functional differences in the mesolimbic-mesocortical systems and related areas mediate the impulsivity-related behavioral traits that distinguish the Roman rat lines/ strains, including differential sensitivity to the effects of (and preference for) drugs of abuse (for reviews, see Charnay et al, 1995; Driscoll et al, 1980; Fattore et al, 2009; Fernández-Teruel et al, 2002a; Giorgi et al, 1994, 2003, 2007; Guitart-Masip et al, 2006a, b, 2008a, b; Kulikov et al, 1995; Lecca et al, 2004). Collectively, the neurochemical findings 
reported in these studies suggest a relatively increased function (or tone) of DA and 5-HT neurotransmission in the RHA rat line/strain relative to RLA animals. However, regarding the DA system, these between-strain differences have been clearly identified after different challenges/ treatments or stressors as being far from consistent in their direction when evaluated under baseline conditions. In fact, the common finding has been the absence of RHA vs RLA differences in DA function (outflow, as measured by microdialysis) under baseline conditions (Giorgi et al, 2007, for review).

The neurochemical results of this study revealed betweenstrain differences in monoamine systems in the striatum and nucleus accumbens under basal conditions. Compared with the RLA-I rats, RHA-I animals showed 5-HT hyperactivity based on increased levels of 5-HT and the metabolite 5-HIAA in both brain areas, and an increased 5-HIAA/5-HT turnover ratio in the nucleus accumbens. These results would appear to agree with the neurochemical evidence described above regarding 5-HT differences between RHA and RLA (outbred) rats. In addition, this is the first time that between-strain differences in NE levels has been reported in Roman rats, RHA-I showed increased NE levels in both brain areas compared with RLA-I rats. Conversely, although there were no between-strain differences in DA or its metabolites (DOPAC and HVA), RHA-I rats showed a relative (but significant) basal hypoactivity in the dopaminergic system as suggested by a decreased (DOPAC + HVA)/DA turnover ratio in the striatum and nucleus accumbens. This finding appears to be partially at variance with previous results (Driscoll et al, 1990) obtained with the RHA/Verh and RLA/Verh outbred rat lines (from which the present RHA-I/RLA-I inbred strains were derived in 1993); however, that previous study is not completely comparable to the present work because the investigators measured DA turnover by determining DOPAC disappearance after pargyline (MAO inhibitor) treatment.

We have found significant relations between neurochemistry findings and impulsivity measures only in RHA-I rats. The individual data of the RHA-I showed in nucleus accumbens that reduced levels of 5-HIAA were related with impulsive responding in the 5-CSRT task; and in the striatum increased levels of DA, DOPAC, and 5-HT activity ratio were associated with preference for larger delayed rewards (or decreased impulsivity). These results seem to be consistent with in vivo studies that associate decreasing 5HT with impulsive responding (Harrison et al, 1997; Winstanley et al, 2004). However, post-mortem studies revealed no relation between DA and 5-HT and their metabolites in nucleus accumbens and dorsal striatum with impulsive premature responses (Dalley et al, 2002; Puumala and Sirviö, 1998). Although, high impulsive subjects in the 5-CSRT task showed increased DA activity in other brain areas: the anterior cingulate, prelimbic, and infralimbic cortices (Dalley et al, 2002). The reason for these discrepancies may be due to procedural differences, the most prominent being from the selection of a specific animal strain. In summary, the RHA-I rats, characterized as more impulsive than RLA-I, revealed higher levels of 5-HT and lower levels of DA under basal conditions; however, correlations found in RHA-I rats have shown that lower levels of 5-HT were associated with an increase in impulsivity while higher levels of DA were associated with a decrease in impulsivity. These apparent contradictory results could be related to the complexity of the 5-HT and DA involvement in impulsivity (Dalley et al, 2008 for review). In vivo neurochemical studies may allow a clearer understanding of the role of monoamines in the impulsive phenotype showed in RHA-I rats.

The current results provide a pattern of consistent behavioral and neurochemical differences between the RHA-I and RLA-I rat strains, in which RHA-I rats showed a high impulsive phenotype across different measures for impulsive behavior as well as in an OCD model. High levels of impulsivity are a cause and/or consequence of many disabling psychopathological disorders in children and adults, including OCD, attention-deficit hyperactivity disorder, schizophrenia, antisocial behavior, and addictive behaviors. Thus, neurobehavioral studies targeting exploration of the different forms of impulsivity may have a broader relevance for future treatments of these psychopathologies. This study is the first to directly characterize these Roman rat strains as a valid model for studying the different aspects of impulsive behaviors and implies their usefulness as animal models of human psychopathological disorders.

\section{ACKNOWLEDGEMENTS}

This work was supported by project SEJ2006-15226-C0201/ PSIC, granted by the Ministerio de Ciencia e Innovación, subdirección general de proyectos de investigación and by FEDER funds. AF-T and AT receive support from EURATools (European Commission Contract no. LSHG-CT2005-019015), PSI2009-10532, and 2009-SGR-51. We thank A Cañete and L Ruedas for their invaluable and continuous help.

\section{DISCLOSURE}

The authors declare no conflict of interest.

\section{REFERENCES}

Aguilar R, Gil L, Gray JA, Driscoll P, Flint J, Dawson GR et al. (2003). Fearfulness and sex in F2 Roman rats: males display more fear though both sexes share the same fearfulness traits. Physiol Behav 78: 723-732.

American Psychiatric Association (2000). Diagnostic and Statistical Manual of Mental Disorders 4th edn, text revision. American Psychiatric Press: Washington, DC.

Anderson KG, Woolverton WL (2005). Effects of clomipramine on self-control choice in Lewis and Fischer 344 rats. Pharmacol Biochem Behav 80: 387-393.

Annetrude JG, de Mooij-van Malsen A, Berend Olivier AB, Martien JHK (2008). Behavioural genetics in mood and anxiety: a next step in finding novel pharmacological targets. Eur J Pharmacol 585: 436-440.

Aubry JM, Bartanusz V, Driscoll P, Schulz P, Steimer T, Kiss Jz (1995). Corticotropin-releasing factor and vasopressin mRNA levels in Roman high- and low-avoidance rats: response to openfield exposure. Neuroendocrinology 61: 89-97.

Broadhurst PL, Bignami G (1965). Correlative effects of psychogenetic selection: a study of the Roman high and low avoidance strains of rats. Behav Res Ther 3: 273-280. 
Cardinal RN, Pennicott DR, Sugathapala CL, Robbins TW, Everitt BJ (2001). Impulsive choice induced in rats by lesions of the nucleus accumbens core. Science 292: 2499-2501.

Cardona D, López-Grancha M, López-Crespo G, Nieto-Escamez F, Sánchez-Santed F, Flores P (2006). Vulnerability of long-term neurotoxicity of chlorpyrifos: effect on schedule-induced polydipsia and a delay discounting task. Psychopharmacology 189: 47-57.

Carli M, Robbins TW, Evenden JL, Everitt BJ (1983). Effects of lesions to ascending noradrenergic neurones on performance of a 5-choice serial reaction task in rats; implications for theories of dorsal noradrenergic bundle function based on selective attention and arousal. Behav Brain Res 9: 361-380.

Carrasco J, Márquez C, Nadal R, Tobeña A, Fernández-Teruel A, Armario A (2008). Characterization of central and peripheral components of the hypothalamus-pituitary-adrenal axis in the inbred Roman rat strains. Psychoneuroendocrinology 33: 437-445.

Chakroun N, Doron J, Swendsen J (2004). Substance use, affective problems and personality traits: test of two association models. Encephale 30: 564-569.

Charnay Y, Steimer T, Huguenin C, Driscoll P (1995). $\left[{ }^{3} \mathrm{H}\right]$ Paroxetine binding sites: brain regional differences between two psychogenetically selected lines of rats. Neurosci Res Comm. 16: 29-35.

Corda MG, Piras G, Lecca D, Fernández-Teruel A, Driscoll P, Giorgi O (2005). The psychogenetically selected Roman rat lines differ in the susceptibility to develop amphetamine sensitization. Behav Brain Res 157: 147-156.

Crabbe JC (2002). Genetic contributions to addiction. Annu Rev Psychol 53: 435-462.

Dalley JW, Mar AC, Economidou D, Robbins TW (2008). Neurobehavioral mechanisms of impulsivity: fronto-striatal systems and functional neurochemistry. Pharmacol Biochem Behav 90: 250-260.

Dalley JW, Theobald DE, Pereira EA, Li PM, Robbins TW (2002). Specific abnormalities in serotonin release in the prefrontal cortex of isolation-reared rats measured during behavioural performance of a task assessing visuospatial attention and impulsivity. Psychopharmacology 164: 329-340.

Daruna JH, Barnes PA (1993). A neurodevelopmental view of impulsivity. In: McCown WG, Johnson JL, Shure MB (eds). The Impulsive Client: Theory, Research and Treatment. American Psychological Association: Washington DC. pp 23-37.

Driscoll P, Bättig K (1982). Behavioural, emotional and neurochemical profiles of rats selected for extreme differences in active, two-way avoidance performance. In: Lieblich I (ed). Genetics of the Brain. Elsevier: Amsterdam. pp 95-123.

Driscoll P, Dedek J, D’Angio M, Claustre Y, Scatton B (1990). A genetically-based model for divergent stress response: behavioral, neurochemical and hormonal aspects. In: Pliska V, and Stranzinger G (eds). Farm Animals in Biomedical Research, vol. 5. Verlay Paul Parey: Hamburg, pp 97-107.

Driscoll P, Dedek J, Martin JR, Baettig K (1980). Regional 5-HT analysis in Roman high- and low-avoidance rats following MAO inhibition. Eur J Pharmacol 68: 373-376.

Driscoll P, Escorihuela RM, Fernández-Teruel A, Giorgi O, Schwegler H, Steimer TH et al. (1998). Genetic selection and differential stress responses: the Roman lines/strain of rats. In: Csermely P (ed). Stress of Life: From Molecules to Man. New York Academy of Sciences: New York. pp 501-510.

Driscoll P, Fernández-Teruel A, Corda MG, Giorgi O, Steimer T (2009). Some guidelines for defining personality differences in rats. In: Kim YK (ed). Handbook of Behavior Genetics. Springer: New York. pp 281-300.

Escorihuela RM, Fernández-Teruel A, Gil L, Aguilar R, Tobeña A, Driscoll P (1999). Inbred Roman high- and low-avoidance rats: differences in anxiety, novelty-seeking, and shuttlebox behaviors. Physiol Behav 67: 19-26.
Escorihuela RM, Fernández-Teruel A, Tobeña A, Langhans W, Bättig K, Driscoll P (1997). Labyrinth exploration, emotional reactivity, and conditioned fear in young Roman/Verh inbred rats. Behav Genet 27: 573-578.

Escorihuela RM, Tobeña A, Driscoll P, Fernández-Teruel A (1995). Effects of training, early handling, and perinatal flumazenil on shuttle box acquisition in Roman low-avoidance rats: toward overcoming a genetic deficit. Neurosci Biobehav Rev 19: 353-367.

Evenden JL (1999). Varieties of impulsivity. Psychopharmacology 146: 413-421.

Evenden JL, Ryan CN (1996). The pharmacology of impulsive behaviour in rats: the effects of drugs on response choice with varying delays of reinforcement. Psychopharmacology 128: 161-170.

Fattore L, Piras G, Corda MG, Giorgi O (2009). The Roman highand low-avoidance rat lines differ in the acquisition, maintenance, extinction, and reinstatement of intravenous cocaine self-administration. Neuropsychopharmacology 34: 1091-1101.

Falk JL (1961). Production of polydipsia in normal rats by an intermittent food schedule. Science 133: 195-196.

Fernández-Teruel A, Escorihuela RM, Castellano B, González B, Tobeña A (1997). Neonatal handling and environmental enrichment effects on emotionality, novelty/reward seeking, and agerelated cognitive and hippocampal impairments: focus on the Roman rat lines. Behav Genet 27: 513-526.

Fernández-Teruel A, Escorihuela RM, Núñez JF, Gomà M, Driscoll P, Tobeña A (1992). Early stimulation effects on novelty-induced behavior in two psychogenetically-selected rat lines with divergent emotionality profiles. Neurosci Lett 137: 185-188.

Fernández-Teruel A, Driscoll P, Gil L, Aguilar R, Tobeña A, Escorihuela RM (2002a). Enduring effects of environmental enrichment on novelty seeking, saccharin and ethanol intake in two rat lines (RHA/Verh and RLA/Verh) differing in incentiveseeking behavior. Pharmacol Biochem Behav 73: 225-231.

Fernández-Teruel A, Giménez-Llort L, Escorihuela RM, Gil L, Aguilar R, Steimer $\mathrm{T}$ et al. (2002b). Early-life handling stimulation and environmental enrichment: are some of their effects mediated by similar neural mechanisms? Pharmacol Biochem Behav 73: 233-245.

Ferre P, Fernández-Teruel L, Escorihuela RM, Driscoll P, Corda MG, Giorgi O et al. (1995). Behavior of the Roman/very highand low-avoidance rat lines in anxiety tests: relationship with defecation and self-grooming. Physiol Behav 58: 1209-1213.

Gentsch C, Lichtsteiner M, Feer H (1991). Genetic and environmental influences on reactive and spontaneous locomotor activities in rats. Experientia 47: 998-1008.

Gilpin NW, Badia-Elder NE, Elder RL, Stewart RB (2008). Schedule-induced polydipsia in lines of rats selectively bred for high and low ethanol preference. Behav Genet 38: 515-524.

Giménez-Llort L, Cañete T, Guitart-Masip M, Fernández-Teruel A, Tobeña A (2005). Two distinctive apomorphine-induced phenotypes in the Roman high- and low-avoidance rats. Physiol Behav 86: $458-466$.

Giorgi O, Lecca D, Piras G, Driscoll P, Corda MG (2003). Dissociation between mesocortical dopamine release and fearrelated behaviours in two psychogenetically selected lines of rats that differ in coping strategies to aversive conditions. Eur $J$ Neurosci 17: 2716-2726.

Giorgi O, Orlandi M, Escorihuela RM, Driscoll P, Lecca D, Corda MG (1994). GABAergic and dopaminergic transmission in the brain of Roman high-avoidance and Roman low-avoidance rats. Brain Res 638: 133-138.

Giorgi O, Piras G, Corda MG (2007). The psychogenetically selected Roman high- and low-avoidance rat lines: a model to study the individual vulnerability to drug addiction. Neurosci Biobehav Rev 31: 148-163.

Gould TD, Gottesman II (2006). Psychiatric endophenotypes and the development of valid animal models. Genes Brain Behav 5: 113-119. 
Guitart-Masip M, Giménez-Llort L, Fernández-Teruel A, Cañete T, Tobeña A, Ogren SO et al. (2006a). Reduced ethanol response in the alcohol-preferring RHA rats and neuropeptide mRNAs in relevant structures. Eur J Neurosci 23: 531-540.

Guitart-Masip M, Johansson B, Cañete T, Fernández-Teruel A, Tobeña A, Terenius L et al. (2008a). Regional adaptations in PSD-95, NGFI-A and secretogranin gene transcripts related to vulnerability to behavioral sensitization to amphetamine in the Roman rat strains. Neuroscience 151: 195-208.

Guitart-Masip M, Johansson B, Fernández-Teruel A, Cañete T, Tobeña A, Terenius L et al. (2006b). Divergent anatomical pattern of D1 and D3 binding and dopamine- and cyclic AMPregulated phosphoprotein of $32 \mathrm{kDa}$ mRNA expression in the Roman rat strains: implications for drug addiction. Neuroscience 142: 1231-1243.

Guitart-Masip M, Johansson B, Fernández-Teruel A, Tobeña A, Giménez-Llort L. (2008b). Divergent effect of the selective D3 receptor agonist pd-128,907 on locomotor activity in Roman high- and low-avoidance rats: relationship to NGFI-A gene expression in the Calleja islands. Psychopharmacology 196: 39-49.

Harrison AA, Everitt BJ, Robbins TW (1997). Central 5-HT depletion enhances impulsive responding without affecting the accuracy of attentional performance: interactions with dopaminergic mechanisms. Psychopharmacology 133: 329-342.

Kosten TA, Ambrosio E (2002). HPA axis function and drug addictive behaviors: insights from studies with Lewis and Fischer 344 inbred rats. Psychoneuroendocrinology 27: 35-69.

Kreek MJ, Nielsen DA, Butelman ER, LaForge KS (2005). Genetic influences on impulsivity, risk taking, stress responsivity and vulnerability to drug abuse and addiction. Nat Neurosci 8: 1450-1457.

Kulikov A, Castanon N, Morméde P, Chaouloff F (1995). Cerebral tryptophan hydroxylase activity, and $5-\mathrm{HT}_{1 \mathrm{~A}}$ receptor, $5-\mathrm{HT}_{2 \mathrm{~A}}$ receptor, and 5-HT transporter binding in grouped and isolated Roman RHA an RLA rats: relationships with behaviours in two models of anxiety. Psychopharmacology 121: 385-395.

Laakso A, Mohn AR, Gainetdinov RR, Caron MG (2002). Experimental genetic approaches to addiction. Neuron 36: 213-228.

Lecca D, Piras G, Driscoll P, Giorgi O, Corda MG (2004). A differential activation of dopamine output in the shell and core of the nucleus accumbens is associated with the motor responses to addictive drugs: a brain dialysis study in Roman high- and low-avoidance rats. Neurochopharmacology 46: 688-699.

López-Aumatell R, Blázquez G, Gil L, Aguilar R, Cañete T, Giménez-Llort L et al. (2009a). The Roman high- and lowavoidance rat strains differ in fear-potentiated startle and classical aversive conditioning. Psicothema 21: 27-32.

López-Aumatell R, Vicens-Costa E, Guitart-Masip M, MartinezMembrives E, Valdar W, Johannesson M et al. (2009b). Unlearned anxiety predicts learned fear: a comparison among heterogeneous rats and the Roman rat strains. Behav Brain Res 202: $92-101$.

López-Grancha M, López-Crespo G, Sánchez-Amate MC, Flores $\mathrm{P}$ (2008). Individual differences in schedule-induced polydipsia and the role of gabaergic and dopaminergic systems. Psychopharmacology 197: 487-498.

Machado-Vieira R, Kapczinski F, Soares JC (2004). Perspectives for the development of animal models of bipolar disorder. Prog Neuropsychopharmacol Biol Psychiatry 28: 209-224.

Mittleman G, Valenstein E (1984). Ingestive behavior evoked by hypothalamic stimulation and schedule-induced polydipsia are related. Science 224: 415-417.

Mittleman G, Valenstein E (1985). Individual differences in non-regulatory ingestive behavior and catecholamine systems. Brain Res 348: 112-117.
Mittleman G, Van Brunt CL, Matthews DB (2003). Scheduleinduced ethanol self-administration in DBA/2J and C57BL/6J mice. Alcohol Clin Exp Res 27: 918-925.

Moreno M, Cañadas F, Cardona D, Flores P, Suñol C, Campa L et al. (2008). Long-term monoamine alteration in striatum and nucleus accumbens after acute Chlorpyrifos exposure. Toxicol Letters 176: 162-167.

Moreno M, Ruedas L, Flores P (2009). Vulnerability differences in binge-eating behavior after schedule induced polydipsia. Behav Pharmacol 20: S38.

Piazza PV, Mittleman G, Deminiere JM, Le Moal M, Simon H (1993). Relationship between schedule-induced polydipsia and amphetamine intravenous self-administration. Individual differences and role of experience. Behav Brain Res 55: 185-193.

Platt B, Beyer CE, Schechter LE, Rosenzweig-Lipson S (2008). Schedule-induced polydipsia: a rat model of obsessive-compulsive disorder. Curr Protoc Neurosci 9: 1-7.

Puumala T, Sirviö J (1998). Changes in activities of dopamine and serotonin systems in the frontal cortex underlie poor choice accuracy and impulsivity of rats in an attention task. Neuroscience 83: 489-499.

Robbins TW (2002). The 5-choice serial reaction time task: behavioural pharmacology and functional neurochemistry. Psychopharmacology 163: 362-380.

Robinson ES, Eagle DM, Economidou D, Theobald DE, Mar AC, Murphy ER et al. (2009). Behavioural characterisation of high impulsivity on the 5-choice serial reaction time task: specific deficits in 'waiting' versus 'stopping'. Behav Brain Res 196: 310-316.

Rosas JM, Callejas-Aguilera JE, Escarabajal MD, Gomez MJ, de la Torre L, Agüero A et al. (2007). Successive negative contrast effect in instrumental runway behaviour: a study with Roman high- (RHA) and Roman low- (RLA) avoidance rats. Behav Brain Res 185: 1-8.

Rosenzweig-Lipson S, Sabb A, Snack G, Mitchell P, Lucki I, Malberg JE et al. (2007). Antidepressant-like effects of the novel, selective, $5-\mathrm{HT}_{2 \mathrm{C}}$ receptor agonist WAY-163909. Psychopharmacology 192: 159-170.

Sánchez-Cardoso P, Higuera-Matas A, Martín S, Miguéns M, Del Olmo N, García-Lecumberri C et al. (2009). Strain differences between Lewis and Fischer 344 rats in the modulation of dopaminergic receptors after morphine self-administration and during extinction. Neuropharmacology 57: 8-17.

Semenova S, Markou A (2007). The effects of the mGluR5 antagonist MPEP and the mGluR2/3 antagonist LY341495 on rats' performance in the 5-choice serial reaction time task. Neuropharmacology 52: 863-872.

Semenova S, Stolerman IP, Markou A (2007). Chronic nicotine administration improves attention while nicotine withdrawal induces performance deficits in the 5-choice serial reaction time task in rats. Pharmacol, Biochem Behav 87: 360-368.

Sher KJ, Bartholow BD, Wood MD (2000). Personality and substance use disorders: a prospective study. J Consult Clin Psychol 68: 818-829.

Steimer T, Driscoll P (2003). Divergent stress responses and coping styles in psychogenetically selected Roman high-(RHA) and low-(RLA) avoidance rats: behavioural, neuroendocrine and developmental aspects. Stress 6: 87-100.

Steimer T, Driscoll P (2005). Inter-individual vs line/strain differences in psychogenetically selected Roman High-(RHA) and Low-(RLA) Avoidance rats: neuroendocrine and behavioural aspects. Neurosci Biobehav Rev 29: 99-112.

Toscano CA, Kameyama M, Garcia-Mijares M, Silva MT, Santarem EM (2008). Relationship between ethanol and sucrose selfadministration and schedule-induced polydipsia. Pharmacol Biochem Behav 90: 586-589. 
van Gaalen MM, Brueggeman RJ, Bronius PF, Schoffelmeer AN, Vanderschuren LJ (2006). Behavioral disinhibition requires dopamine receptor activation. Psychopharmacology 187: 73-85.

Winstanley C, Dalley J, Theobald D, Robbins T (2004). Fractionating impulsivity: contrasting effects of central 5-HT depletion on different measures if impulsive behaviour. Neuropsychopharmacology 29: 1331-1343.

Winstanley CA, Eagle DM, Robbins TW (2006). Behavioral models of impulsivity in relation to ADHD: translation between clinical and preclinical studies. Clin Psychol Rev 26: 379-395.
Woods A, Smith C, Szewczak M, Dunn RW, Cornfeldt M, Corbett R (1993). Selective serotonin re-uptake inhibitors decrease scheduleinduced polydipsia in rats: a potential model for obsessive compulsive disorder. Psychopharmacology 112: 195-198.

Woods-Kettelberger AT, Smith CP, Corbett R, Szewczak MR, Roehr JE, Bores GM et al. (1996). Besipirdine (HP 749) reduces schedule-induced polydipsia in rats. Brain Res Bull 41: 125-130. Zeier H, Baettig K, Driscoll P (1978). Acquisition of DRL-20 behavior in male and female, Roman high- and low-avoidance rats. Physiol Behav 20: 791-793. 